\title{
CALIBRACION DE UN MODELO ECONOMÉTRICO PARA EL ANÁLISIS DE LA FRECUENCIA DE ACCIDENTES DE TRÁNSITO CON PEATONES
}

\section{CALIBRATING A ECONOMETRIC MODEL FOR THE ANALYSIS OF THE FREQUENCY OF TRAFFIC ACCIDENTS WITH PEDESTRIAN}

\author{
Ing. Yefry Quintero Avendaño ${ }^{\mathrm{a}}$, MSc Andrea S. Arevalo Tamara ${ }^{\mathrm{b}}$, MSc Thomas E. Guerrero
} Barbosa $^{\mathrm{c}}$
${ }^{a}$ Universidad Francisco de Paula Santander Ocaña,Vía Acolsure Sede el Algodonal, Ocaña - Norte de Santander, Colombia, Ye.fry100@hotmail.com
${ }^{\mathrm{b}}$ Universidad Francisco de Paula Santander Ocaña, Grupo de Investigación GRUCITE, Vía Acolsure Sede el Algodonal, Ocaña - Norte de Santander, Colombia, Ing.andrea_arevalo@hotmail.es
${ }^{\mathrm{c}}$ Universidad Francisco de Paula Santander Ocaña, Grupo de Investigación GRUCITE, Vía Acolsure Sede el Algodonal, Ocaña - Norte de Santander, Colombia, teguerrerob@ufpso.edu.co

\begin{abstract}
Resumen: En la Ciudad de Ocaña Norte de Santander en los últimos años se ha presentado una alta tasa de accidentalidad donde se ven involucrados peatones tanto de tipo lesión como de muertes. Una de las principales causas de accidentes donde se ven involucrados peatones es principalmente la falta de espacios peatonales como anchos de andén, cruces peatonales y otros como imprudencia por parte de los conductores (vehículos, motocicletas), falta de señalización entre otras. Para esta investigación se tuvieron en cuenta los accidentes ocurridos durante los años 2007 a 2014 en la ciudad de Ocaña, Norte de Santander, donde se vieron involucrados peatones. Se evaluaron las variables que influyen en esta accidentalidad en los tramos más críticos en zona urbana. Entre las variables que se encontraron significantes están el ancho de la calzada, el transito promedio diario, Número de intersecciones en el tramo, la velocidad (percentil 15), el sentido de la vía y el uso de suelo.
\end{abstract}

Palabras clave: Accidentalidad, Binomial Negativo, Frecuencia, Peatón, Poisson.

Abstract: In Ocaña in recent years there has been a high rate of accidents involving pedestrians where both types of injury and deaths. One of the main causes of accidents in which pedestrians are involved is mainly the lack of pedestrian spaces and widths platform, crosswalks and others like recklessness by drivers (cars, motorcycles), lack of signals among others. For this investigation accidents during the years 2007-2014 in Ocaña, where pedestrians were involved. The variables that influence accidents in the most critical sections 
in urban areas were evaluated. Among the variables that were found significant are the width of the roadway, the TPD (average daily traffic), number of intersections in the stretch, the speed (15th percentile), the direction of the track and land use.

\section{INTRODUCCIÓN}

Todas las políticas sostenibles de transporte en la actualidad, se están orientando para descongestionar los sistemas viales de nuestras ciudades promoviendo los vehículos compartidos, gestionando los estacionamientos, incentivando la flexibilidad horaria laboral, incorporando el teletrabajo, impulsando el uso de la bicicleta, usando el sistema público masivo y estimulando los desplazamientos a pie; respecto a este último modo de viaje, en algún momento las personas lo han incorporado como parte de su rutina de viaje, sin embargo las estadísticas muestran que uno de los actores más afectados por accidentalidad son los peatones. Reportes de (World Health Organization, 2013) evidencian que cada año a nivel mundial los peatones constituyen 270.000 muertes a causa de la accidentalidad vial, cifras preocupantes dado que estos actores viales representan el $22 \%$ del total de defunciones por accidentes de tránsito y en algunos países alcanza cifras alarmantes de hasta el 66\%.

Para en caso de Colombia (Instituto Nacional de Medicina Legal y Ciencias Forenses, 2015), los principales grupos vulnerables en términos de mortalidad debido a accidentes de tránsito lo constituyen los motociclistas (45,52\%), peatones $(27,41 \%)$ y ciclistas $(5,36 \%)$. En el primer estudio de caracterización del comportamiento de peatones en Colombia realizado por el (Fondo de Prevención Vial, 2011) se reporta que los peatones representan 3 de cada 10 víctimas de accidentes de tránsito. Otras conclusiones del mismo estudio arrojan que: el $61 \%$ de los colombianos son caminantes habituales, en promedio, los colombianos caminan 69 minutos al día, divididos en 3 o 4 trayectos. La mayoría de peatones considera que es inofensivo violar las normas de tránsito, aunque el $71 \%$ dice que las normas hay que cumplirlas, el $25 \%$ aduce que hay momentos en que se pueden incumplir. Los peatones toman decisiones sin planear recorridos generando comportamientos incorrectos. El 67\% de los entrevistados se calificaron como buenos peatones y entre un 38\% a un $40 \%$ aceptó tener comportamientos descuidados $\mathrm{o}$ infractores en las vías.

Debido a estos antecedentes, el Estado Colombiano comenzó a definir medidas claras y concretas para reducir los efectos de las cusas e impactos negativos asociados a la accidentalidad y por ende todos los actores viales implicados entre ellos los peatones, particularmente el (Ministerio de Transporte, 2014) mediante la Resolución 2273 del 6 de agosto de 2014 adoptó el Plan Nacional de Seguridad Vial, el cual dentro de sus objetivos específicos contempla reducir la mortalidad en un $18 \%$ del usuario tipo peatón por accidentes de tránsito para el año 2021. Dado este contexto, la presente investigación se enfocó en estimar un modelo econométrico a partir de aproximaciones tipo Poisson y Binomial Negativo para determinar la frecuencia en accidentes de tránsito con peatones cuyos datos provienen de una base de datos de registros de accidentes con peatones involucrados entre los años 2007 hasta 2014. 
Es amplia la literatura que ha abordado la estimación de frecuencias de accidentes en peatones a partir de modelos como Poisson (Geyer, Raford, Pham, \& Ragland, 2006) (Ye, Zhang, \& Lord, 2013) y Binomial Negativo (Guevara, Washington, \& Oh, 2004) (Haque, Chin, \& Huang, 2010) (Tulu, Washington, Haque, \& King, 2015), sin embargo existe una reciente preocupación ya que en paises en desarrollo la identificación de los factores que influyen en la accidentalidad de peatones no es clara debido a la deficiencia de registros específicos y características que rodean este tipo de accidentes (Tulu, Washington, Haque, \& King, 2015). Respecto a las variables que influyen en la modelación de este tipo de eventos, existe evidencia que se ha enfocado en estudiar este fenómeno especialmente en las intersecciones (Bonneson, Pratt, \& Songchitruksa, 2011) (Miranda-Moreno, Morency, \& ElGeneidy, 2011) (Hamidun, Ishak, \& Endut, 2013) debido a que es en estos sitios donde con frecuencia hay conflictos de cruce entre peatones y flujos vehiculares; igualmente investigaciones reportan la relevancia de considerar los flujos de peatones (Zegeer, Stewart, Huang, \& Lagerwey, 2000) (Harwood, y otros, 2008), variable no considerada en la presente investigación. Por otro lado, variables relacionadas con los volúmenes vehiculares (Harwood, y otros, 2008) (Sharma \& Landge, 2012) (Tulu, Washington, Haque, \& King, 2015), geometría de las vías y el entorno (Sharma \& Landge, 2012) (Tulu, Washington, Haque, \& King, 2015), condiciones climáticas (Eisenberg, 2004).

En líneas generales la investigación se distribuye de la siguiente forma: en la sección XX se explica la naturaleza y procedencia de los datos además del enfoque metodológico utilizado.
Seguidamente en la sección XX se muestra la estimación de los modelos con su respectivo análisis. Finalmente en la sección XX se relacionan las diferentes conclusiones del estudio.

\section{METODOLOGÍA}

Para el análisis de la accidentalidad con peatones en la ciudad de Ocaña se utilizaron las distribuciones tradicionales Poisson y Binomial Negativa.

\subsection{Poisson}

El modelo tradicional y punto de partida para el análisis de datos de accidentes es el Poisson (Miaou, 1993).

Para esta distribución los datos de accidentes se caracterizan mejor como ensayos de Bernoulli con probabilidades desiguales de accidentes que varían entre los conductores, los vehículos, las carreteras, y las condiciones ambientales (Lord, 2006). Debido a la baja probabilidad de un accidente y el gran número de ensayos, estos ensayos de Bernoulli pueden ser aproximados como ensayos de Poisson. Por lo tanto la distribución tradicional de Poisson se considera que es el punto de partida para el análisis de datos de accidentes de tránsito. Según Lee \& Mannering, 2002 el número de accidentes $n_{i j}$ para un segmento en particular $i$ durante un periodo $j$ está dado por:

$$
P\left(n_{i j}\right)=\frac{\exp \left(-\lambda_{i j}\right) \lambda_{i j}^{n_{i j}}}{n_{i j} !}
$$

Dónde:

$P\left(n_{i j}\right)=$ es la probabilidad de que ocurran $n$ accidentes en una sección de carretera $i$ en un periodo de tiempo $j$. 
$\lambda_{i j}=$ es el valor previsto de $n_{i j}$, que es el número esperado de accidentes por año en un tramo,

$$
\lambda_{i j}=\exp \left(\beta X_{i j}\right)
$$

Para una sección de carretera $i$ en el periodo de tiempo $j, \beta$ es un vector de coeficiente de regresión desconocida que puede estimarse por métodos de máxima verosimilitud estándar (Greene, 1994).

$X_{i j}=$ es un vector de variables que describen la sección o característica geométrica de la vía, características ambientales y otras condiciones relevantes de la carretera que afectan la frecuencia de los accidentes como por ejemplo la presencia de berma, estado del pavimento, longitud del tramo, tipo de área, número de curvas, TPD, entre otras.

Una limitación de utilizar el modelo de regresión de Poisson, que es bien conocido en la literatura estadística (por ejemplo Cox, 1983 Dean y Lawless, 1989; Luis M., Liping Fu, 2006), consiste en que la varianza de los datos está restringido a ser igual a la media de regresión estimado a partir de los datos. Sin embargo, se han presentado casos donde la varianza de los datos fue mayor que lo que indica el modelo Poisson (por ejemplo: Dean y Lawless 1989), (Miaou, 1993).

\subsection{Modelo Binomial Negativo (BN):}

Para subsanar la más notoria limitación que se ha encontrado en el modelo de Poisson (sobredispersion), se opta por la distribución $\mathrm{BN}$ que se deriva de la ecuación 2, de tal manera que para cada tramo i y tiempo $\mathrm{j}$, tiene la siguiente forma (Washington, et al., 2003): $\lambda_{i j}=E X P\left(\beta X_{i j}+\varepsilon_{i j}\right)$

Dónde:

$\operatorname{EXP}\left(\varepsilon_{i j}\right)=$ es un término de error de distribución gamma con media 1 y varianza $\alpha^{2}$. La adición de este periodo permite a la varianza diferenciarse de la media de la siguiente forma (Washington, et al., 2003):

$\operatorname{VAR}\left[n_{i j}\right]=E\left[n_{i j}\right]\left[1+\alpha E\left[n_{i j}\right]\right]=$
$E\left[n_{i j}\right]+\alpha E\left[n_{i j}\right]^{2}$

La distribución BN tiene la forma (Washington, et al., 2003):

$$
\begin{aligned}
& P\left(n_{i j}\right)= \\
& \frac{\Gamma\left((1 / \alpha)+n_{i j}\right)}{\Gamma(1 / \alpha) n_{i j} !}\left(\frac{1 / \alpha}{(1 / \alpha)+\lambda_{i j}}\right)^{1 / \alpha}\left(\frac{\lambda_{i j}}{(1 / \alpha)+\lambda_{i j}}\right)^{n_{i j}}
\end{aligned}
$$

Dónde:

$\Gamma()=$. es una función gamma.

La media y la varianza de la variable aleatoria de BN está dada por (Geedipally, 2008):

$E\left(n_{i j}\right)=\lambda_{i j}$

$\operatorname{Var}\left(n_{i j}\right)=\lambda_{i j}+\alpha \lambda_{i j}^{2}$

Es de tener en cuenta que $\alpha \rightarrow 0$, la varianza de los accidentes es igual a la media y este modelo converge en el modelo estándar de regresión de Poisson.

\subsection{Datos}

En esta investigación se utilizaron datos de accidentalidad con peatones ocurridos en 
las principales vías de la ciudad de Ocaña, Norte de Santander durante los años 2012 al 2014.

Para la recopilación de los datos de accidentalidad se contó con el apoyo y colaboración de la policía nacional y el cuerpo de bomberos de la ciudad, quienes son los encargados de realizar el registro de cada uno de los siniestros ocurridos en la ciudad.

Se seleccionaron los tramos que se consideran críticos para evaluar la accidentalidad con peatones donde la variable dependiente de la modelación corresponde a el número de accidentes de cada trayecto y las variables dependientes hacen referencia a características de la vía, el entorno y del tránsito.

En total se seleccionaron 14 tramos con longitudes variables entre 300 metros hasta $4 \mathrm{~km}$. Dependiendo de las características de la vía, los tramos fueron lo más homogéneos posibles entre sí.

A continuación en la Tabla 2.2.1 se muestra las características de las variables continuas relacionadas con la vía.

\begin{tabular}{|c|c|c|c|c|}
\hline & $\begin{array}{l}\text { Ancho de } \\
\text { calzada }\end{array}$ & No. Intersecciones & $\begin{array}{c}\text { No. } \\
\text { Carriles }\end{array}$ & Sentidos \\
\hline Descripción & $\mathrm{mt}$ & cantidad & cantidad & cantidad \\
\hline Máximo & 4084,26 & 35 & 4 & 1 \\
\hline Mínimo & 374,85 & 6 & 2 & 0 \\
\hline $\begin{array}{l}\text { Desviación } \\
\text { estándar }\end{array}$ & 971,3 & 7,8 & 0,5 & 0,4 \\
\hline \multicolumn{5}{|c|}{ Fuente: Elaboración propia } \\
\hline \multicolumn{5}{|c|}{$\begin{array}{l}\text { Las variables como tipo de pavimento, } \\
\text { funcionalidad, demarcación, presencia de } \\
\text { separador, uso de suelo, presencia de } \\
\text { andén, iluminación y presencia de } \\
\text { señalización vertical se tomaron como }\end{array}$} \\
\hline
\end{tabular}

variables mudas tomando valores de 0 y 1 según el caso.

\section{RESULTADOS}

Para el análisis de accidentalidad en la ciudad, se calibraron 2 modelos, uno con distribución Poisson y otro con distribución Binomial negativo donde se puede apreciar la influencia de las variables en la ocurrencia de accidentes donde se ven involucrados peatones.

\subsection{Modelo Poisson}

Como se observa en la ¡Error! No se encuentra el origen de la referencia. las variables incluidas en este modelo dieron como resultado los signos esperados, además, también se aprecia que la variable más significativa es la cantidad de motocicletas por tramo. Esto se debe principalmente a las altas velocidades a las que circulan los conductores de estos vehículos, la facilidad de transitar en espacios reducidos, la invasión de espacios peatonales, entre otras imprudencias con la que se caracterizan en la ciudad.

La presencia de vehículos pesados y de intersecciones aumenta la probabilidad de ocurrencia de accidentes de tránsito donde resulten muertes o heridos los peatones de las vías. En los tramos donde los anchos de carril son mayores y en las vías doble sentido se presentan más accidentes que ponen en riesgo la vida de los transeúntes.

Referente al uso de suelo, es de esperarse que en las zonas residenciales se presenten más accidentes con peatones por la cantidad de personas sobre la vía.

Contrario a muchos estudios, la velocidad no resultó ser un causante de este tipo de accidentes, concordando con el estudio realizado por Sasidharan \& Menéndez en el año 2014 donde evaluaron la severidad de los accidentes con peatones en Suiza, donde determinaron que esta variable no 
influye en los accidentes con peatones debido a que en zonas urbanas los vehículos tienden a ir más despacio por la cantidad de controles de tráfico que existen sobre este tipo de zona.

La variable tipo de pavimento es un caso que poco ha sido estudiado. En esta investigación se incluyó en los modelos dando como resultado que en los pavimentos rígidos tiende a ocurrir más accidentes con peatones. Esto puede explicarse específicamente para Ocaña puesto que la mayoría de las calles urbanas de la ciudad están conformadas por este tipo de pavimento.

\subsection{Modelo Binomial Negativo}

Para realizar una comparación entre los resultados de cada distribución, en este modelo se incluyó las mismas variables que para el modelo Poisson. Al igual que el modelo anterior, los signos fueron los esperados pero se observa que la significancia de las variables disminuyo considerablemente. Los resultados se muestran en la

Tabla 3.1.

En los resultados se observa que la cantidad de motos aún sigue siendo la variable más influyente seguida del número de intersecciones por ser un punto crítico de paso peatonal

Al realizar una comparación entre los dos modelos calibrados, se puede concluir que el mejor ajuste se lleva a cabo con la distribución Poisson por la significancia de las variables y comparando la logverosimilitud.

Tabla 3.1 Resultados de la calibración con distribución Poisson y BN

\begin{tabular}{|c|c|c|c|c|c|c|c|c|c|c|}
\hline \multicolumn{6}{|c|}{ POISSON } & \multicolumn{5}{|c|}{ BINOMIAL NEGATIVO } \\
\hline \multirow{2}{*}{$\begin{array}{l}\text { Variables } \\
\text { Vehículos } \\
\text { pesados }\end{array}$} & \multirow{2}{*}{$\begin{array}{c}\text { Coeficiente } \\
0,00\end{array}$} & \multirow{2}{*}{$\begin{array}{c}\mathbf{Z} \\
1,49\end{array}$} & \multirow{2}{*}{$\begin{array}{l}\mathbf{P}>|\mathbf{z}| \\
0,136\end{array}$} & \multicolumn{2}{|c|}{$\begin{array}{c}\text { [95\% coef. } \\
\text { Interval] }\end{array}$} & \multirow{2}{*}{$\begin{array}{c}\text { Coeficiente } \\
0,00\end{array}$} & \multirow{2}{*}{$\begin{array}{c}\mathbf{Z} \\
1,55\end{array}$} & \multirow{2}{*}{$\begin{array}{l}\mathbf{P}>|\mathbf{z}| \\
0,121\end{array}$} & \multicolumn{2}{|c|}{$\begin{array}{c}\text { [95\% coef. } \\
\text { Interval] }\end{array}$} \\
\hline & & & & 0,000 & 0,003 & & & & 0,000 & 0,005 \\
\hline Uso de suelo & 0,97 & 2,03 & 0,042 & 0,034 & 1,914 & 1,22 & 1,45 & 0,147 & $-0,427$ & 2,868 \\
\hline $\begin{array}{r}\text { Tipo de } \\
\text { pavimento }\end{array}$ & 0,77 & 1,86 & 0,063 & $-0,041$ & 1,59 & 1,15 & 1,58 & 0,115 & $-0,28$ & 2,587 \\
\hline Sentido & 2,32 & 3,04 & 0,002 & 0,825 & 3,81 & 2,72 & 1,98 & 0,047 & 0,031 & 5,42 \\
\hline $\begin{array}{r}\text { Ancho de } \\
\text { carril }\end{array}$ & 0,82 & 3,27 & 0,001 & 0,327 & 1,304 & 0,81 & 1,73 & 0,083 & $-0,105$ & 1,715 \\
\hline $\begin{array}{r}\text { Numero de } \\
\text { intersecciones }\end{array}$ & 0,53 & 3,31 & 0,001 & 0,021 & 0,084 & 0,048 & 2,00 & 0,046 & 0,000 & 0,095 \\
\hline Motocicletas & 0,00 & 4,16 & 0,000 & 0,000 & 0 & 0,00 & 2,39 & 0,017 & 0,000 & 0,000 \\
\hline _cons & $-6,75$ & $-3,01$ & 0,003 & $-11,15$ & $\begin{array}{c}- \\
2,348\end{array}$ & $-7,091$ & $\begin{array}{c}- \\
1,71\end{array}$ & 0,087 & $\begin{array}{c}- \\
15,212\end{array}$ & 1,030 \\
\hline $\begin{array}{r}\text { Log- } \\
\text { likelihood }\end{array}$ & & & 1061 & & & & & 13406 & & \\
\hline
\end{tabular}

Fuente: Elaboración propia 


\section{CONCLUSIONES}

Esta investigación se centró en realizar una comparación entre las distribuciones utilizadas para evaluar las variables influyentes en la accidentalidad que involucran muertes y heridos de peatones en la ciudad de Ocaña, Norte de Santander. Se calibraron dos modelos, el primero con distribución Poisson, siendo esta la más común entre este tipo de análisis y en muchos casos, la más adecuada. Los resultados de esta calibración muestran los signos convenientes y las variables revelan una significancia apropiada. Además de esto, presenta la log-verosimilitud mayor entre las dos distribuciones.

El segundo, con distribución Binomial Negativo. En este caso, disminuye la significancia de las variables pero conservando los signos adecuados.

De lo anterior, se dedujo que el mejor modelo calibrado fue el Poisson.

$\mathrm{Al}$ igual que Sasidharan \& Menéndez en el año 2014, la velocidad no fue una variable influyente en la accidentalidad con peatones cuya explicación se da debido a la gran cantidad de controladores de tráfico (señales, reductores, semáforos, etc.) que existen dentro de las zonas urbanas de la ciudad.

La variable más influyente en la accidentalidad con peatones en la ciudad es la cantidad de motos presentes en el trafico vehicula. En Ocaña el número de motocicletas que circulan es bastante elevado y son el tipo de vehículo que más se ve involucrado en accidentes de tránsito pues no tienen un control adecuado de los requisitos mínimos de un motorizado.

\section{BIBLIOGRAFIA}

Bonneson, J., Pratt, M., \& Songchitruksa, P. (2011). Development of Guidlines for Pedestrian Safety Treatments at Signalised Intersection.

Eisenberg. (2004). The mixed effects of precipitation on traffic crashes. Accident Analisys and Prevention , 36, 637-647.

Fondo de Prevención Vial. (2011). ¿Todos los Caminantes son peatones? Bogotá D.C.

Geedipally, S. R. (2008). Examining the application of conwaymaxwellpoisson models for analyzing traffic crash data.

Geyer, J., Raford, N., Pham, T., \& Ragland, D. R. (2006). Safety in numbers. Data from Oakland, California. Transportation Research Record: Journal of the Transportation Research Board, 1982, 150-154.

Guevara, F. L., Washington, S. P., $\&$ Oh, J. (2004). Forecasting crashes at the planning level: Simultaneous negative binomial crash model applied in Tucson, Arizona. Transportation Research Record.

Hamidun, R., Ishak, S. Z., \& Endut, I. R. (2013). Assessing Pedestrian Crossing Risk at Signalised Intersection. International Journal of Emerging Technology and Advanced Engineering , 3 (1), 31-35.

Haque, M. M., Chin, H. C., \& Huang, H. (2010). Applying Bayesian hierarchical models to examine motorcycle crashes at signalized intersections. 
Accident Analisys and Prevention, 42 (1), 203-212.

Harwood, D. W., Torbic, D. J., Gilmore, D. K., Bokenkroger, C. D., Dunn, J. M., Zegeer, C. V., y otros. (2008). Pedestrian Safety Prediction Methodology. Document 129: Phase III, NCHRP.

Instituto Nacional de Medicina Legal y Ciencias Forenses. (2015). Forensis 2014 Datos para la vida. Bogotá D.C.

Lee, J., \& Mannering, F. (2002). Impact of roadside features on the frequency and severity of run-off-riadway accidents: an empirical analysis. Accident Analysis and Prevention.

Lord, D. (2006). Modeling motor vehicle crashes using Poisson-gamma models: Examining the effects of low sample mean values and small sample size on the estimation of the fixed dispersion parameter. Accident Analysis and Prevention.

Miaou, S. (1993). The relationship between truck accidents and geometric design of road sections: Poisson versus Negative Binoal regressions. Accident Analysis and Prevention.

Ministerio de Transporte. (2014). Plan Nacional de Seguridad Vial 20112021 (Resolución 2273 del 6 de Agosto de 2014). Bogotá D.C.

Miranda-Moreno, L. F., Morency, P., \& El-Geneidy, A. M. (2011). The link between built environment, pedestrian activity and pedestrian-vehicle collision occurrence at. Aciident Analisys and Prevention , 43 (5), 1624-1634.

Sasidharan, L., \& Menéndez, M. (2014). Partial proportional odds model An alternate choice analyzing pedestrian crash injury severities. Accident Analysis and Prevention. 72, 330-340.

Sharma, A. K., \& Landge, V. S. (2012). Pedestrian accident prediction model for rural road. International Journal of Science and Advanced Technology, 2 (8), 66-72.

Tulu, G. S., Washington, S., Haque, M. M., \& King, M. J. (2015). Investigation of pedestrian crashes on two-way two-lane rural roads in Ethiopia. Accident Analysis and Prevention , 78, 118-126.

Washington, S. P., Karlaftis, M. G., \& Mannering,F. L. (2003). Statistical and econometric methods for transportation data analysis. Boca Raton, London, New York, Washington D.C.

World Health Organization. (2013). Global status report on road safety 2013: supporting a decade of action. Department of Violence \& Injury Prevention \& Disability, Geneva.

Ye, Z., Zhang, Y., \& Lord, D. (2013). Goodness-of-fit testing for accident models with low means. Accident Analisys and Prevention , 61, 78-86.

Zegeer, C. V., Stewart, J. R., Huang, H. H., \& Lagerwey, P. A. (2000). Safety Effects of Marked versus Unmarked Crosswalks at Uncontrolled Locations: Executive Summary and Recommended Guidelines 